Regular Article

\title{
Influence of La substitution on local structural and photoluminescence properties of $\mathrm{SrTiO}_{3}:$ Pr phosphor
}

\author{
Lucas Angelini Deltreggia ${ }^{a}$, Maria Inês Basso Bernardi ${ }^{\mathrm{b}}$, Alexandre Mesquita ${ }^{\mathrm{a}, *}$ \\ a Institute of Geosciences and Exact Sciences, São Paulo State University (Unesp), Rio Claro, SP, Brazil \\ b São Carlos Institute of Physics, University of São Paulo, USP, São Carlos, SP, Brazil
}

\section{A R T I C L E I N F O}

\section{Article history:}

Received 15 June 2018

Received in revised form 10 July 2018

Accepted 15 July 2018

Available online 27 July 2018

\begin{abstract}
A B S T R A C T
$\mathrm{Pr}^{3+}$-doped perovskite-based $\mathrm{SrTiO}_{3}$ phosphors have been developed as a potential red phosphor. In this paper, we report the substitution of $\mathrm{La}^{3+}$ ions for $\mathrm{Sr}^{2+}$ ions in order to promote $\mathrm{Sr}$ vacancies to further enhance photoluminescence. Given that the La content increases, an enhancement in the intensity of all emissions is observed, mainly for ${ }^{1} \mathrm{D}_{2}-{ }^{3} \mathrm{H}_{4}$ and ${ }^{3} \mathrm{P}_{0}-{ }^{3} \mathrm{H}_{6}$ transitions. This enhancement of photoluminescence emissions is associated with disorder in the lattice caused by La incorporation. Raman measurements and X-ray absorption near edge structure (XANES) spectra at the Ti K-edge identify this disorder as a function of the La content.
\end{abstract}

(c) 2018 Acta Materialia Inc. Published by Elsevier Ltd. All rights reserved.
Luminescent materials, or phosphors, typically consist of a host material and an activator and are widely used in optoelectronic devices, such as lasers, solar concentrators, vacuum fluorescent displays, medical radiology equipment (including scintillators), field emission displays and light-emitting diodes [1-3]. Host materials for phosphors include sulfides, metaloxides, oxy-halides and perovskites [2,4-6]. Concerning activators, remarkable progress has been observed in the development of rare earth ions as luminescent centers in different semiconductors due to their narrow emission bands ( $f-f$ interactions) and high internal quantum efficiencies. Promising applications exist in the field of photonics as solid state lasers and optoelectronic devices [6]. Among luminescent materials with rare earth ion activators, $\mathrm{Pr}^{3+}$-doped inorganic materials are well known for their promising emission properties, in particular as red phosphors [1]. The three red emission lines arise from different origins of the $4 \mathrm{f}^{2}-4 \mathrm{f}^{2}$ transitions, i.e. ${ }^{1} \mathrm{D}_{2}-{ }^{3} \mathrm{H}_{4}(603 \mathrm{~nm})$, ${ }^{3} \mathrm{P}_{0^{-}}{ }^{3} \mathrm{H}_{6}(617 \mathrm{~nm})$ and ${ }^{3} \mathrm{P}_{0^{-}}{ }^{3} \mathrm{~F}_{2}(650 \mathrm{~nm})$, and occur in addition to two green emission lines $\left({ }^{3} \mathrm{P}_{1}-{ }^{3} \mathrm{H}_{5}(530 \mathrm{~nm})\right.$ and $\left.{ }^{3} \mathrm{P}_{0}-{ }^{3} \mathrm{H}_{5}(547 \mathrm{~nm})\right)$ and one blue-green emission line $\left({ }^{3} \mathrm{P}_{0}-{ }^{3} \mathrm{H}_{4}(491 \mathrm{~nm})\right)[1,7]$.

$\mathrm{Pr}^{3+}$-doped perovskite-based $\mathrm{SrTiO}_{3}\left(\mathrm{SrTiO}_{3}: \mathrm{Pr}\right)$ phosphors have been developed as potential red phosphors for devices operating with low-energy electron excitation and present better physical and chemical stability compared to conventional sulfide compounds [8]. Nevertheless, $\mathrm{SrTiO}_{3}$ :Pr phosphors emit weak luminescence since the $\mathrm{Pr}^{3+}$ ions, which act as activators and emit the red luminescence, cannot directly absorb the radiation used for excitation [8]. However, the introduction of metal cations of different valences into the $\mathrm{SrTiO}_{3}$ matrix can enhance the emission via a number of mechanisms. For example, lattice defects created by $\mathrm{Al}^{3+}$ incorporation can absorb the excitation

\footnotetext{
* Corresponding author.

E-mail address: mesquita@rc.unesp.b (A. Mesquita).
}

radiation, acting as sensitizers, and transfer it to the $\mathrm{Pr}^{3+}$ ions, resulting in their characteristic red emission [8].Moreover, the doping of $\mathrm{SrTiO}_{3}$ : $\mathrm{Pr}^{3+}$ powders with divalent metal ions $\left(\mathrm{Mg}^{2+}\right.$ and $\left.\mathrm{Zn}^{2+}\right)$ forms impurity energy levels near the bottom of the conduction band that provide more efficient energy transfer from the matrix to the $\operatorname{Pr}^{3+}$ ions $[8,9]$. Furthermore, replacement of $\mathrm{Sr}$ with a pair of $\mathrm{Li}^{+}$and $\mathrm{Na}^{+}$ions is believed to facilitate the formation of hole traps, increasing the probability of recombination of electrons with captured holes, thus raising the luminescence intensity [9]. Enhancement of luminescence is also observed as a result of the introduction of trivalent metal ions, such as $\mathrm{Al}^{3+}, \mathrm{Ga}^{3+}$ and $\mathrm{In}^{3+}$, substituting for $\mathrm{Ti}^{4+}$, explained by the compensation for the $\mathrm{Pr}^{3+}$ charge in the site of $\mathrm{Sr}^{2+}$ ions and the higher degree of crystallinity $[8,9]$.

Substitutions of monovalent cations for $\mathrm{Sr}^{2+}$ and trivalent cations for $\mathrm{Ti}^{4+}$ are used in order to achieve charge compensation. A strontium vacancy can, however, be promoted to achieve charge compensation as $\mathrm{Pr}^{3+}$ is typically thought to occupy a $\mathrm{Sr}^{2+}$ site. In this case, the formula $\mathrm{Sr}_{1-3 \times / 2} \mathrm{Pr}_{x} \mathrm{TiO}_{3}$ can be adopted and charge compensation occurs by $x / 2$ formula units of $\mathrm{Sr}$ vacancies per formula unit of $\mathrm{Pr}^{3+}$. Hence, in this paper we report the substitution of $\mathrm{Sr}^{2+}$ ions by trivalent $\mathrm{La}$ ions in order to promote more $\mathrm{Sr}$ vacancies than $x / 2$ to compensate the additional positive charge. In principle, the disordered structure caused by $\mathrm{Sr}$ vacancies can originate from the lower symmetry at $\mathrm{Pr}^{3+}$ sites, which can mix opposite parity into $4 f$ configurational levels, subsequently increasing the ${ }^{1} \mathrm{D}_{2}-{ }^{3} \mathrm{H}_{4}$ transition probabilities of $\mathrm{Pr}^{3+}$ ions and further enhance the luminescence of $\operatorname{Pr}^{3+}[10]$.

In this study, $\mathrm{Sr}_{1-3(x+y) / 2} \mathrm{Pr}_{x} \mathrm{La}_{y} \mathrm{TiO}_{3}$ (SLPT) samples $(x=0.01, y=$ 0.05 (SLPT5) and 0.10 (SLPT10)) were prepared by the polymeric precursor method, which has proven to be an effective procedure for the preparation of $\mathrm{SrTiO}_{3}(\mathrm{ST})$ powders [11]. Strontium carbonate $\mathrm{SrCO}_{3}$ (99\%, Aldrich), titanium isopropoxide $\left[\mathrm{Ti}\left(\mathrm{OC}_{3} \mathrm{H}_{7}\right)_{4}\right]$ (98\%, Aldrich), 
praseodymium oxide $\mathrm{Pr}_{2} \mathrm{O}_{3}$ (99.9\% Alfa Aesar), lanthanum nitrate hexahydrate (99\%, Aldrich), ethylene glycol, $\mathrm{C}_{2} \mathrm{H}_{6} \mathrm{O}_{2}$ (99.5\%, Synth) and citric acid $\mathrm{C}_{6} \mathrm{H}_{8} \mathrm{O}_{7}$ (99.5\%, Synth) were used as raw materials. Titanium citrate was formed by titanium isopropoxide dissolution in a citric acid aqueous solution with constant stirring. The citrate solution was stirred at $70{ }^{\circ} \mathrm{C}$ to obtain a clear homogeneous solution. Stoichiometric quantities of strontium carbonate, lanthanum nitrate hexahydrate and praseodymium nitrate $\left(\mathrm{Pr}_{2} \mathrm{O}_{3}\right.$ was dissolved with $\mathrm{HNO}_{3}$ to convert into $\mathrm{Pr}$ $\left.\left(\mathrm{NO}_{3}\right)_{3}\right)$ were added to the Ti citrate solution. After cation homogenization in the solutions, $\mathrm{C}_{2} \mathrm{H}_{6} \mathrm{O}_{2}$ was added to promote a polyesterification reaction. The citric acid/ethylene glycol mass ratio was fixed at 60/40. As the preparation involves polymeric network formation, the samples were heated at $400{ }^{\circ} \mathrm{C}$ for $3 \mathrm{~h}$ to eliminate the organic precursors. After pyrolysis, all samples were annealed at $700{ }^{\circ} \mathrm{C}$ for $2 \mathrm{~h}$.

Fig. 1 exhibits X-ray diffraction (XRD) patterns of the SLPT powders. These room-temperature measurements were performed in a RigakuUltima 4 powder diffractometer with geometry $\theta-2 \theta$, with a rotating anode X-ray source ( $\mathrm{Cu}-\mathrm{K} \alpha$ radiation, $\lambda=1.542 \AA$ ) and a scintillation detector. The data were collected with a $0.02^{\circ}$ step size at $5 \mathrm{~s}$ per step. As can be seen in Fig. 1, all samples crystallized and were indexed as cubic perovskite structures with $\mathrm{Pm} 3 \mathrm{~m}$ space group [10]. A small peak can be observed at $\sim 25.2^{\circ}$ and is assigned to the secondary phase of $\mathrm{SrCO}_{3}$ with Pmcn space group [10]. In the $\mathrm{SrTiO}_{3}$ matrix, $\mathrm{Pr}^{3+}$ can substitute for $\mathrm{Sr}^{2+}$ because their ionic radii are almost identical. The ionic radii of $\mathrm{Pr}^{3+}$ in the twelve and six-coordinated state are 1.30

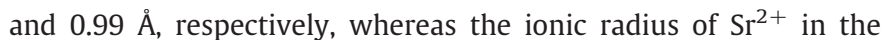
twelve-coordinated state is $1.44 \AA$. Given that the ionic radius of $\mathrm{Ti}^{4+}$ in the six-coordinated state is $0.605 \AA$ [12], and since the samples were prepared considering the stoichiometric $\mathrm{Sr}_{1-3 / 2(\mathrm{x}+\mathrm{y})} \mathrm{Pr}_{\mathrm{x}} \mathrm{La}_{\mathrm{y}} \mathrm{TiO}_{3}$ formula, $\mathrm{Pr}^{3+}$ ions preferentially occupy $\mathrm{Sr}$ instead of Ti sites because of the similar ionic radii.

The local structure of SLPT samples was characterized by X-ray absorption spectroscopy at Ti K-edge measurements. X-ray absorption near edge structure (XANES) spectra give information on the coordination symmetry and the valence of ions incorporated in a solid. The energy of the absorption edge shifts according to the valence of the absorbing ion, since the binding energy of bound electrons rises as the valence increases. Also, the shape of the absorption edge depends on the unfilled local density of states and the coordination symmetry of the absorbing element. X-ray absorption spectra (4966 eV) were collected at the LNLS (National Synchrotron Light Laboratory, Brazil) facility using the D04B-XAS1 beamline. The LNLS storage ring was operated at $1.36 \mathrm{GeV}$ and $100-160 \mathrm{~mA}$. XANES spectra were collected

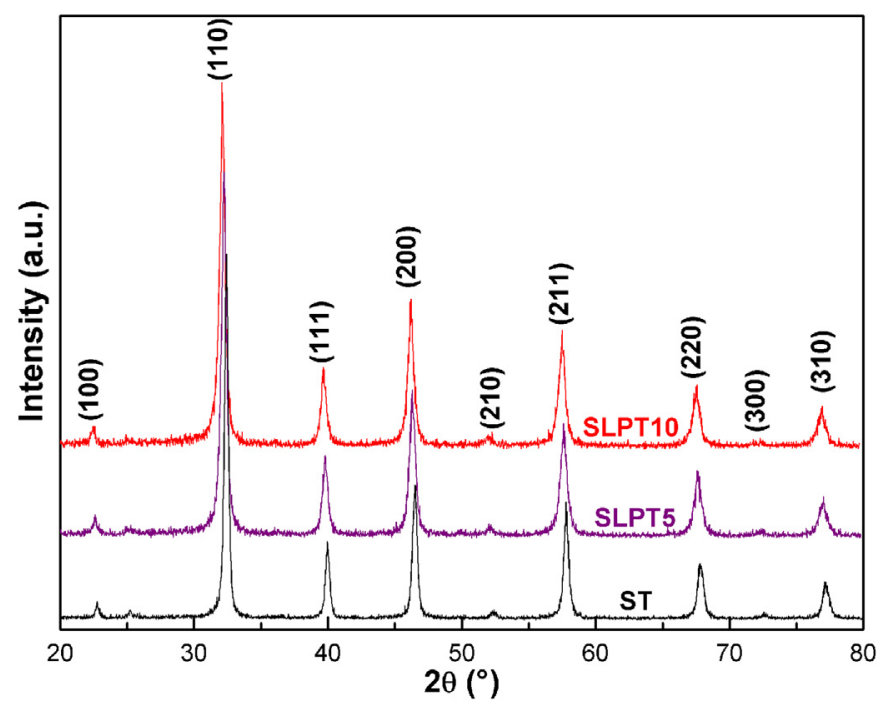

Fig. 1. X-ray diffraction for ST, SLPT5 and SLPT10 samples.

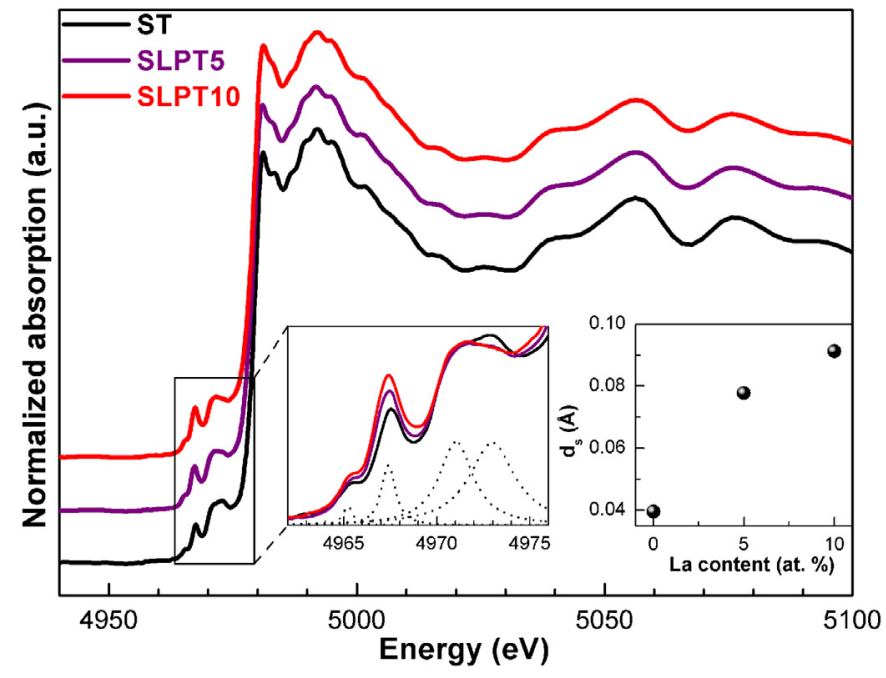

Fig. 2. XANES spectra at the Ti K-edge for ST, SLPT5 and SLPT10 samples. The lower-left inset magnifies the features in the pre-edge region. The lower-right inset shows the static off-center displacement $\left(\mathrm{d}_{\mathrm{s}}\right)$ of $\mathrm{Ti}$ atoms in $\mathrm{TiO}_{6}$ octahedra.

in transmission mode using a $\mathrm{Si}(111)$ channel-cut monochromator at room temperature. The spectra were analyzed through the MultiPlatform Applications for XAFS (MAX) software package [13].

Ti K-edge XANES spectra for SLPT samples are presented in Fig. 2. The lower-left inset of this figure shows in detail the four pre-edge transitions. The pre-edge region of the K-edge XANES spectra of some transition metal oxides is characterized by a pronounced feature occurring several volts before the main rising edge [14]. In transition metal oxides that crystallize in centrosymmetric structures, this pre-edge feature is small or absent, whereas in noncentrosymmetric structures, it can be quite large [14]. The peak at $\sim 4966 \mathrm{eV}$ has pure quadrupole origin due to the $1 s(\mathrm{Ti}) \rightarrow 3 \mathrm{~d}\left(\mathrm{t}_{2 \mathrm{~g}}\right)(\mathrm{Ti})$ transition, whereas the peak at $\sim 4967 \mathrm{eV}$ is due to the $1 s(\mathrm{Ti}) \rightarrow 4 p(\mathrm{Ti})$ transition, including some degree of $1 s(\mathrm{Ti})$ $\rightarrow 3 d\left(e_{g}\right)(\mathrm{Ti})$ quadrupole contribution [10]. The overlapping peaks between 4969 and $4975 \mathrm{eV}$ are assigned to a dipole excitation of $1 \mathrm{~s}$ electrons to $t_{2 g}$ and $e_{g}$ orbitals of the neighboring $\mathrm{TiO}_{6}$ octahedra [10].

As can be seen in Fig. 2, the intensity of the peak at $4967 \mathrm{eV}$ increases as the amount of La increases. This increase has been associated with the fact that Ti ions must be present in the distorted octahedral environment and the area under this peak can be used to determine the off-center displacement in the $\mathrm{TiO}_{6}$ octahedra of the $\mathrm{SrTiO}_{3}$ perovskite structure $[15,16]$. The static displacement $\left(d_{s}\right)$ of Ti ions in the [001] direction was evaluated from the calculation of the area under this peak using a Lorentz function and an empirical relationship $[15,16]$. The results are shown in the lower-right inset of Fig. 2, showing that the amount of $\mathrm{La}^{3+}$ increases with increasing the displacement of the $\mathrm{Ti}$ atom from the centrosymmetric position within the $\mathrm{O}$ octahedron. The small static off-center displacement observed is consistent with previous reports of 0.08 and $0.10 \AA$ [16]. Nonetheless, this displacement indicates a small deviation from cubic symmetryand the La incorporation into the $\mathrm{SrTiO}_{3}$ matrix implies local symmetry breaking. In principle, the Ti off-center displacement should be evaluated more precisely by an EXAFS (extended X-ray absorption fine structure) refinement of the octahedron $\mathrm{TiO}_{6}$ first shell signal. However, the presence of the $\mathrm{La}$ $\mathrm{L}_{\mathrm{III}}$-edge $500 \mathrm{eV}$ above the Ti K-edge limits drastically the EXAFS spatial resolution.

The Raman scattering technique is a useful tool to probe the disorder in the local crystal symmetry [17]. The $\mathrm{SrTiO}_{3}$ cubic perovskite structure with space group Pm $3 m$ only presents the vibration modes $3 \mathrm{~F}_{1 \mathrm{u}}+\mathrm{F}_{2 \mathrm{u}}$ that are not Raman active, and no first-order Raman mode is expected in $\mathrm{SrTiO}_{3}$ at room temperature [18]. Studies of the Raman spectra of $\mathrm{SrTiO}_{3}$ have shown that the Raman modes can be modified, especially 
the activation of the first-order modes, by many factors including strain effects, oxygen vacancies and even external conditions $[17,18]$. Thus, each of the $F_{1 u}$ modes splits into a doubly degenerate $E$ mode and a nondegenerate $A_{1}$ mode, while the $F_{2 u}$ mode splits into $E$ and $B_{1}$ modes [18]. All of the $A_{1}$ and $E$ modes are both Raman and infrared active, while the $\mathrm{B}_{1}$ mode is Raman active. The presence of long-range electrostatic forces further splits each of the $\mathrm{A}_{1}$ and $\mathrm{E}$ modes into transverse optical (TO) and longitudinal optical (LO) modes [18]. Therefore, the fundamental phonon modes of Slater-type $\left(\mathrm{TO}_{1} / \mathrm{LO}_{1}\right)$, Last-type $\left(\mathrm{TO}_{2} / \mathrm{LO}_{2}\right)$ and Axe-type $\left(\mathrm{TO}_{4} / \mathrm{LO}_{4}\right)$, are probably present [17]. The lowest frequency $\mathrm{TO}_{1} / \mathrm{LO}_{1}$ modes arise due to $\mathrm{B}$-ion motions against oxygen vibrations, the intermediate frequency $\mathrm{TO}_{2} / \mathrm{LO}_{2}$ modes originate from A-ion vibrations and the oxygen vibrations in $\mathrm{BO}_{6}$ octahedra give rise to the highest frequency $\mathrm{TO}_{4} / \mathrm{LO}_{4}$ in the $\mathrm{ABO}_{3}$ crystal lattice [17].

Fig. 3 shows the Raman spectra for SLPT samples. These spectra were measured at room temperature with a MonoVista CRS Raman spectrometer (S\&I). The samples were irradiated with a laser beam focused by an Olympus microscope. For the excitation in backscattering geometry, the $633 \mathrm{~nm}$ line of a $35 \mathrm{~mW}$ He-Ne laser was used. The modes in the spectrum of the ST sample are assigned as follows: $\mathrm{TO}_{1}\left(149 \mathrm{~cm}^{-1}\right), \mathrm{TO}_{2}$ $\left(177 \mathrm{~cm}^{-1}\right), \mathrm{TO}_{3}\left(289 \mathrm{~cm}^{-1}\right), \mathrm{LO}_{2}\left(482 \mathrm{~cm}^{-1}\right), \mathrm{TO}_{4}\left(545 \mathrm{~cm}^{-1}\right), \mathrm{TO}$ $\left(730 \mathrm{~cm}^{-1}\right)$ and $\mathrm{LO}_{4}\left(795 \mathrm{~cm}^{-1}\right)$. The peak observed at $1074 \mathrm{~cm}^{-1}$ is related to the $\mathrm{SrCO}_{3}$ phase, in agreement with our XRD measurements [19]. Because the $\mathrm{La}^{3+}$ ions are incorporated in $\mathrm{SrTiO}_{3}$ matrix, the intensity of $\mathrm{TO}_{1}, \mathrm{TO}_{3}$ and $\mathrm{TO}$ modes show a small increase, indicating that Ti sites are not affected by La addition. This result is in agreement with the XANES results, which show a small off-center displacement of Ti atoms as a function of La content. However, the intensity of $\mathrm{TO}_{2}$ and $\mathrm{LO}_{4}$ modes show a substantial increase with La content. Because the $\mathrm{TO}_{2}$ mode originates from $\mathrm{A}$-ion vibration in the $\mathrm{ABO}_{3}$ structure, this increase is an indication of disorder at $\mathrm{Sr}$ sites due to La substitution and the formation of $\mathrm{Sr}$ vacancies. The behavior of the $\mathrm{LO}_{4}$ mode denotes tilting of $\mathrm{TiO}_{6}$ octahedra due to modified oxygen vibrations resulting from this disorder.

The photoluminescence spectra for SLTP5 and SLTP10 samples are show in Fig. 4. The spectra were measured at room temperature with a Thermal Jarrel-Ash Monospec monochromator and a Hamamatsu R446 photomultiplier. The $350.7 \mathrm{~nm}$ exciting wavelength of a krypton ion laser (Coherent Innova) was used; the output of the laser was maintained at $200 \mathrm{~mW}$. The ${ }^{1} \mathrm{D}_{2}-{ }^{3} \mathrm{H}_{4},{ }^{3} \mathrm{P}_{0}-{ }^{3} \mathrm{H}_{6},{ }^{3} \mathrm{P}_{0}-{ }^{3} \mathrm{~F}_{2},{ }^{3} \mathrm{P}_{1}-{ }^{3} \mathrm{H}_{5}$, ${ }^{3} \mathrm{P}_{0^{-}}{ }^{3} \mathrm{H}_{5}$ and ${ }^{3} \mathrm{P}_{0,1,2}{ }^{3} \mathrm{H}_{4}$ transitions are indexed in the spectra of Fig. 4. As can be seen, the photoluminescence curve of the SLPT5 sample shows blue $\left({ }^{3} \mathrm{P}_{0,1,2}{ }^{3} \mathrm{H}_{4}\right)$ and red $\left({ }^{1} \mathrm{D}_{2}-{ }^{3} \mathrm{H}_{4}\right.$ and $\left.{ }^{3} \mathrm{P}_{0}-{ }^{3} \mathrm{H}_{6}\right)$ emissions

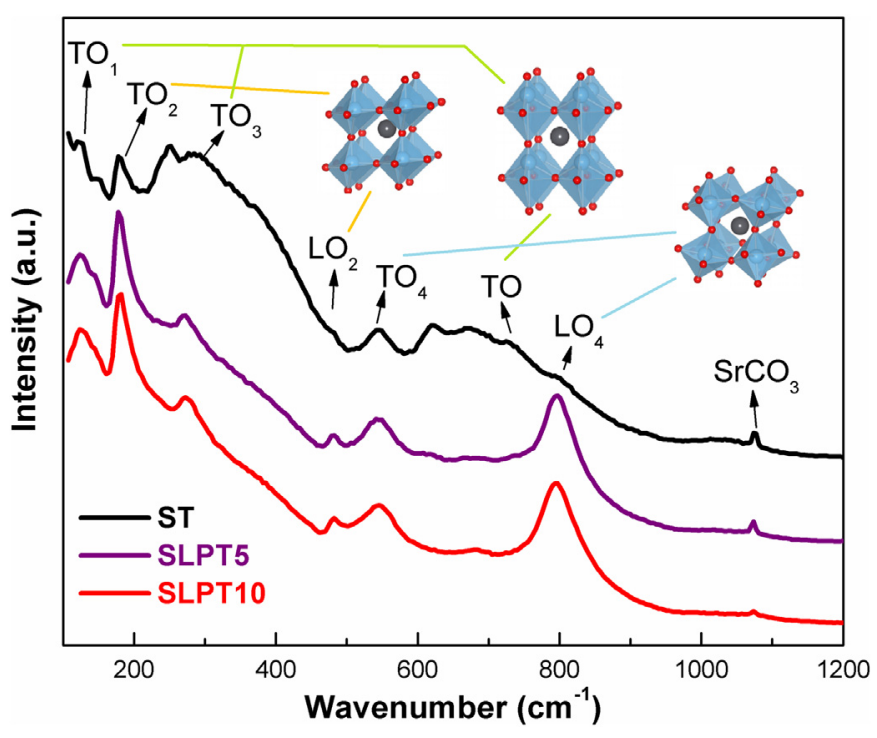

Fig. 3. Raman spectra for ST, SLPT5 and SLPT10 samples.

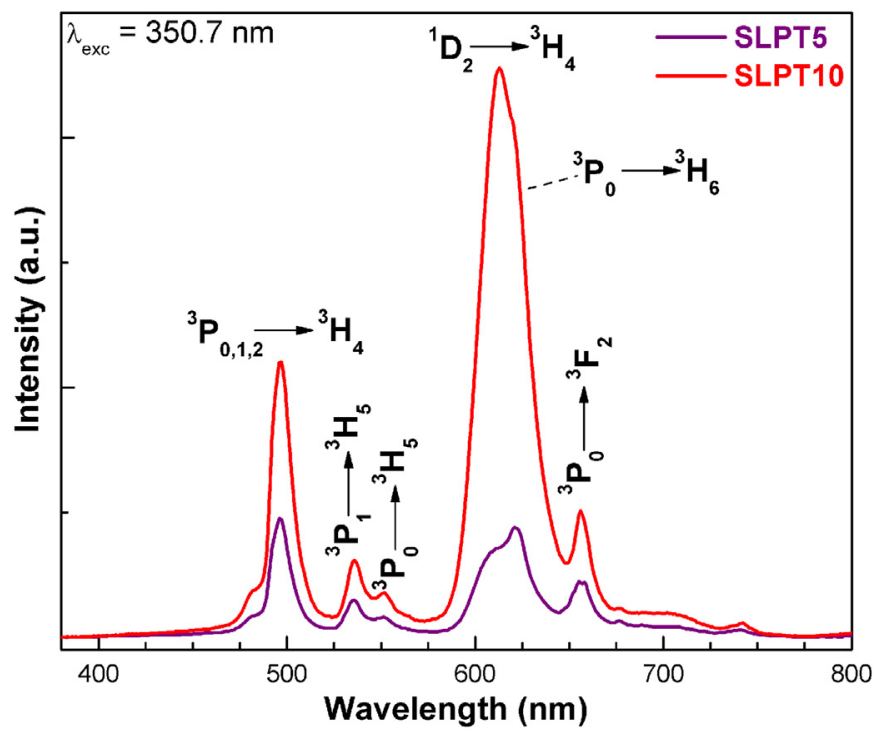

Fig. 4. Photoluminescence spectra for SLPT5 and SLPT10 samples.

with similar intensities. If the combination of the blue to red emissions can be regulated in a controlled manner, white emission from a single host may be realized, which can function as white light-emitting diodes (WLEDs) [1]. The spectrum of the SLPT10 sample shows an enhancement in the intensity of all emissions, mainly for ${ }^{1} \mathrm{D}_{2}-{ }^{3} \mathrm{H}_{4}$ and ${ }^{3} \mathrm{P}_{0}-{ }^{3} \mathrm{H}_{6}$ transitions.

It is well known that the photoluminescence efficiency of a rare earth ion is sensitive to the symmetry and strength of the crystal field and that an increased degree of distortion in the crystal field may enhance their luminescence efficiency [20]. According to Fujiwara et al. [20], in a study comparing $\mathrm{CaTiO}_{3}, \mathrm{SrTiO}_{3}$ and $\mathrm{BaTiO}_{3}$ host lattices, $\mathrm{Pr}^{3+}$ substituted for the $\mathrm{Ca}^{2+}$ site in $\mathrm{CaTiO}_{3}$ : $\mathrm{Pr}$ can emit intense red light without the addition of enhancers, since $\mathrm{CaTiO}_{3}$ has a distorted crystal structure due to the crystal framework constructed by tilted Ti octahedrons [20].Ions of large ionic radius or vacancies substituted for A-site ions tend to induce structural asymmetry in the host, approaching the lower symmetry around $\operatorname{Pr}^{3+}[10]$. In principle, more uneven crystal fields due to the lower symmetry at the $\mathrm{Pr}^{3+}$ sites can mix opposite parity into $4 f$ configurational levels, subsequently increasing the transition probabilities of $\operatorname{Pr}^{3+}$ ions [10].

Thus, we report the substitution of $\mathrm{La}^{3+}$ ions for $\mathrm{Sr}^{2+}$ ions in order to promote Sr vacancies to further enhance the photoluminescence of the $\mathrm{SrTiO}_{3}:$ Pr compound. Because the La content increases, all emission intensities are enhanced, mainly for ${ }^{1} \mathrm{D}_{2}-{ }^{3} \mathrm{H}_{4}$ and ${ }^{3} \mathrm{P}_{0^{-}}{ }^{3} \mathrm{H}_{6}$ transitions. This enhancement of photoluminescence emissions is associated with disorder in the lattice caused by La incorporation. As shown by Raman and XANES measurements, disorder increases with tilting in the titanium octahedron cluster, the latter increasing with La content. Thus, the enhancement of the photoluminescence curves for $\mathrm{SrTiO}_{3}: \mathrm{Pr}$, La samples is associated with disorder in the $\mathrm{SrTiO}_{3}$ lattice caused by La incorporation.

This work was supported by FAPESP (through project 2013/12993-4), CNPq (302743/2014-6) and LNLS (proposal number XAFS1-17750), Brazil. The authors also thank Leandro X. Moreno for Raman measurements.

\section{References}

[1] W. Tang, Y. Sun, M. Yu, X. Liu, Y.Q. Yin, B. Yang, L.M. Zheng, F. Qin, Z.G. Zhang, W.W. Cao, RSC Adv. 5 (35) (2015) 27491-27495.

[2] G.K. Ribeiro, F.S. Vicente, M.I.B. Bernardi, A. Mesquita, J. Alloys Compd. 688 (2016) 497-503. 
[3] C.S. Lewis, H.Q. Liu, J.Y. Han, L. Wang, S.Y. Yue, N.A. Brennan, S.S. Wong, Nanoscale 8 (4) (2016) 2129-2142.

[4] J.L. Huang, L.Y. Zhou, Z.P. Liang, F.Z. Gong, J.P. Han, R.F. Wang, J. Rare Earths 28 (3) (2010) 356-360.

[5] A.A. Othman, M.A. Ali, E.M.M. Ibrahim, M.A. Osman, J. Alloys Compd. 683 (2016) 399-411.

[6] N.P. Bhagya, P.A. Prashanth, R.H. Krishna, B.M. Nagabhushana, R.S. Raveendra, Optik 145 (2017) 678-687.

[7] A. Brenier, I.V. Kityk, J. Appl. Phys. 90 (1) (2001) 232-236.

[8] H. Ryu, B.K. Singh, K.S. Bartwal, M.G. Brik, I.V. Kityk, AcMat 56 (3) (2008) 358-363.

[9] E.A. Bondarenko, A.A. Skomorokhov, N.I. Kargin, A.S. Gusev, S.M. Ryndya, OptSp 118 (5) (2015) 729-734.

[10] H.Q. Sun, Q.W. Zhang, X.S. Wang, Y. Zhang, Ceram. Int. 40 (10) (2014) 15669-15675.

[11] L.F. da Silva, J.C. M'Peko, J. Andres, A. Beltran, L. Gracia, M.I.B. Bernardi, A. Mesquita, E. Antonelli, M.L. Moreira, V.R. Mastelaro, J. Phys. Chem. C 118 (9) (2014) 4930-4940.
[12] R.D. Shannon, AcCrA 32 (SEP 1) (1976) 751-767.

[13] A. Michalowicz, J. Moscovici, D. Muller-Bouvet, K. Provost, J. Phys. Conf. Ser. 190 (2009), 012034.

[14] A. Mesquita, A. Michalowicz, V.R. Mastelaro, J. Appl. Phys. 111 (2012) 104110.

[15] H. Stocker, M. Zschornak, C. Richter, J. Hanzig, F. Hanzig, A. Hinze, K. Potzger, S. Gemming, D.C. Meyer, Scr. Mater. 86 (2014) 1-4.

[16] A.I. Frenkel, D. Ehre, V. Lyahovitskaya, L. Kanner, E. Wachtel, I. Lubomirsky, Phys. Rev. Lett. 99 (21) (2007).

[17] G. Jyothi, K.G. Gopchandran, Dyes Pigments 149 (2018) 531-542.

[18] X.E. Wu, D.J. Wu, X.J. Liu, Solid State Commun. 145 (5-6) (2008) 255-258.

[19] L.F. da Silva, W. Avansi, J. Andres, C. Ribeiro, M.L. Moreira, E. Longo, V.R. Mastelaro, Phys. Chem. Chem. Phys. 15 (29) (2013) 12386-12393.

[20] R. Fujiwara, H. Sano, M. Shimizu, M. Kuwabara, J. Lumin. 129 (2009) 231-237. 